\title{
La movilidad residencial hacia un municipio metropolitano: el caso de Las Rozas de Madrid
}

\author{
Carmen Muguruza Cañas
}

\section{BASES TEORICAS DEL ESTUDIO}

Aunque los geógrafos han incluido desde siempre la movilidad en sus estudios geográficos de la ciudad, sólo recientemente le han dado el énfasis que requiere, dada la complejidad del tema.

La movilidad residencial es contemplada como un producto de las ofertas de viviendas - viviendas nuevas y vacantes en las áreas internas de la ciudad - y de las necesidades y expectativas de los usuarios, que son a su vez producto de la renta, del tamaño de la familia y del estilo de la vida.

La movilidad residencial en las ciudades occidentales es considerable. Sin embargo, todas las evidencias demuestran que las tasas de movilidad residencial son mucho mayores en las ciudades norteamericanas que las europeas. Entre un 7 por 100 y un 12 por 100 de población se mueven dentro de la ciudad y su entorno en los países europeos para cambiar de residencia, esta cifra aumenta al 20 por 100 en las ciudades norteamericanas. Los datos para España ha sido imposible obtenerlos debido a la falta de estudios de este tipo en nuestro país, pero pensamos que la movilidad residencial es más baja que en otros países europeos debido a la importancia que aqui tiene la vivienda en propiedad, lo cual es un freno a la hora de los cambios residenciales. 
En nuestro estudio hemos intentado ver las relaciones que existen entre la movilidad residencial y la estructura urbana existente.

Las relaciones básicas entre movilidad residencial y estructura urbana, las resume Paul $\mathrm{KNOX}^{1}$, en el siguiente cuadro:

\section{CUADRO I. RELACIONES ENTRE MOVILIDAD RESIDENCIAL $Y$} ESTRUCTURA URBANA

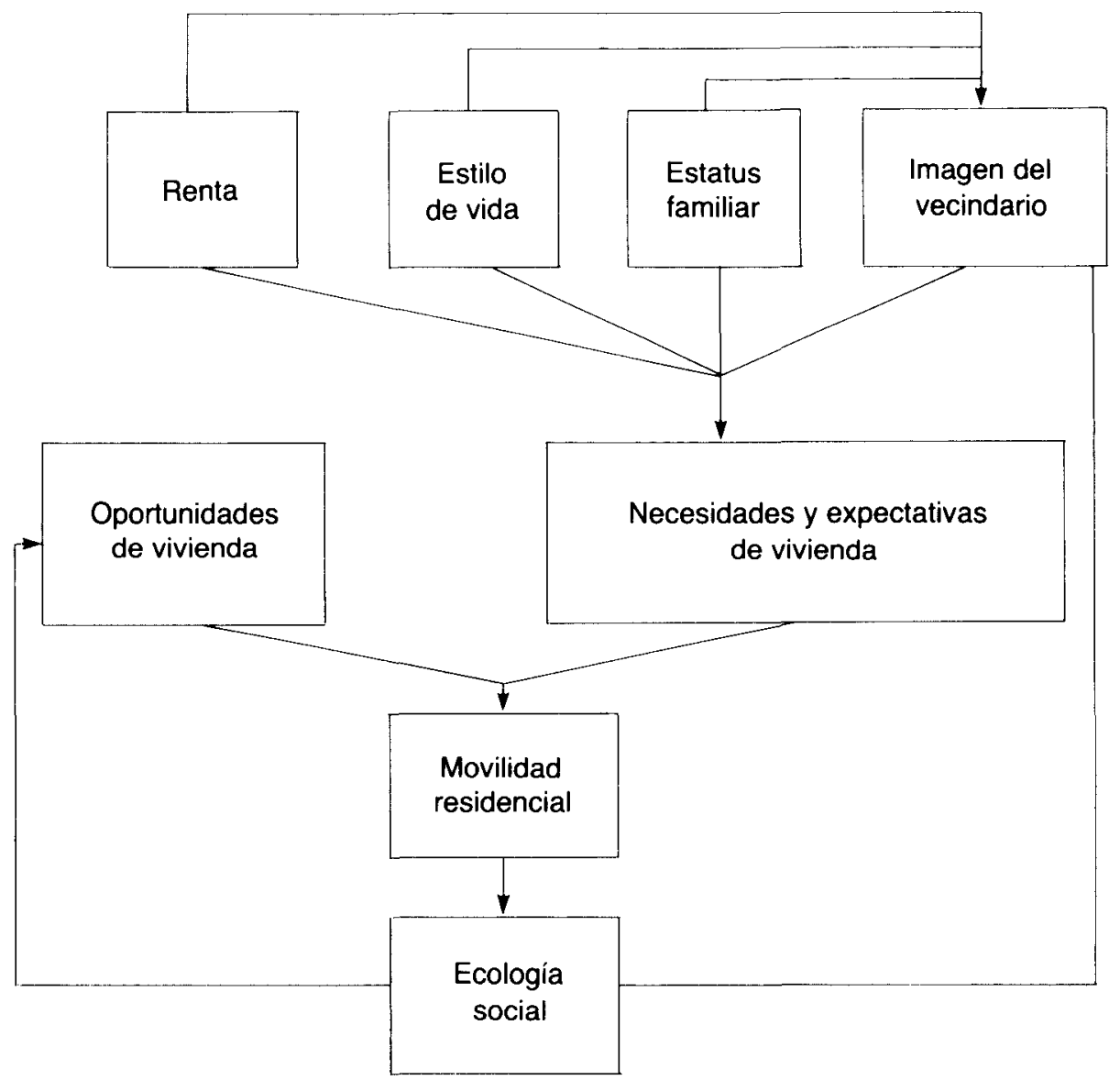

Fuente: Paul Knox: Urban Geography: an introduction.

1 KNox, P. (1982): Urban Social Geography: An Introduction. Longman, N. York, pág. 90. 
Aunque se acepta que los movimientos de un lugar a otro de residencia juegan un importante papel en la formación y definición de las áreas sociales, las relaciones entre movilidad residencial y estructura residencial todavia no están bien definidas. Esto es debido, en parte, a que a pesar de que la movilidad residencial significa un tercio de los movimientos migratorios en las ciudades occidentales, es todavía un hecho reciente. Asimismo, también se debe a la complejidad de estas relaciones, ya que este tipo de migración modela y remodela las estructuras sociales y demográficas de los vecindarios urbanos y está a su vez condicionada por la ecología existente en la ciudad.

De todas formas los estudios sobre movilidad residencial intraurbana son recientes y han desarrollado muy pocos conceptos firmes. Las teorías desarrolladas en el contexto de las migraciones a escala regional no son directamente aplicables. Parece ser que uno de los determinantes generales del nivel de movilidad residencial es económico, durante los períodos de auge económico existe un incremento en la demanda efectiva de nuevas viviendas. Pero son, sobre todo, los cambios en la organización social, en particular los que afectan a la estructura familiar, los que influyen sensiblemente en la movilidad. A más largo plazo, las variaciones en el mercado inmobiliario son también importantes, ya que influyen directamente en la oferta de nuestras viviendas, así como en las facilidades financieras para adquirirlas o alquilarlas. En algunos paises europeos, como España, la expansión de la vivienda en propiedad ha tenido como consecuencia una menor movilidad residencial.

El tema de la movilidad intraurbana ha tenido un tratamiento teórico sobre todo dentro de la geografía de la percepción, y desde postulados behavioristas. Siendo el trabajo de WOLPERT $(1952)^{2}$ uno de los pioneros. Los trabajos de la percepción han puesto su énfasis en la elección de la residencia, tomando como unidad de análisis al individuo. La toma de decisión que lleva a un individuo y a su familia a residir en un lugar determinado de la ciudad refleja una amplia variedad de características personales y urbanas, por lo que evaluar las causas de esa toma de decisión es extremadamente complejo. De hecho, las hipótesis lanzadas sobre los mecanismos de elección de residencia se apoyan, aún hoy en dia, en un escaso volumen de trabajos empíricos.

\footnotetext{
2 WOLPERT, J. (1965): "Behavioral aspects of the decision to migrate", en Papers of Regional Science Association. Vol. 54, pág. 91.
} 
Nosotros en nuestro estudio hemos intentado poner de manifiesto las regularidades espaciales de los movimientos intraurbanos, en la creencia de que si tales regularidades existen nos podrian ayudar a ver las relaciones entre movilidad residencial y estructura social urbana.

Las regularidades en los modelos de migración intraurbana son bastante difíciles de establecer, ya que como hemos dicho, existen muy pocos conceptos firmes en este tema. Sin embargo, los estudios sobre el tema sugieren algunas regularidades en el comportamiento de las personas que emigran: uno de los hallazgos más consistentes de las investigaciones de este tipo es lo referente a la distancia, la mayoria de los estudios han constatado que ésta suele ser corta. La dirección también se ha estudiado aunque parece ser que las conclusiones son menos consistentes. Se reconoce ampliamente una tendencia general de corriente migratoria hacia los suburbios, pero los flujos hacia la ciudad central están menos estudiados. De los movimientos que tiene como fin los suburbios se ha observado que un alto porcentaje $(45 \%)$ se realizan dentro del mismo sector de origen. Las regularidades más significativas en los modelos de movimientos intraurbanos, son las relativas al estatus socioeconómico de origen y destino de las áreas. Así, las personas de un determinado estatus socio-económico suelen ir a residir a áreas de su mismo estatus o mayor, es lo que en este tipo de estudio se ha denominado "afinidad de vecindario". En los estudios de movilidad residencial se ha intentado dar también una clasificación de las razones que existen para el cambio de residencia, distinguiendo entre cambios voluntarios e involuntarios, divorcio, jubilación... Pero todos los investigadores han reconocido que son los cambios en el ciclo familiar la motivación fundamental de los cambios de residencia.

Así, existen distintas necesidades de tamaño de vivienda según el momento del ciclo familiar (matrimonio joven sin hijos, con hijos pequeños, hijos estudiando, personas solas...).

Aunque en España la movilidad residencial intraurbana tiene comparativamente muy poca importancia, es en las zonas suburbanas como las que estamos estudiando donde de alguna manera se producen movimientos residenciales que pueden asimilarse a lo que ocurre en las áreas anglosajonas. Para ello, y una vez consultados los trabajos que existen sobre el tema, intentamos, sistematizar cómo han sido los últimos movimientos inmigratorios que se han producido en Las Rozas, las fuentes de estudio y la organización de los datos lo vamos a exponer en el siguiente punto. 


\section{ANALISIS DE LA METODOLOGIA Y FUENTES UTILIZADAS}

El análisis de los últimos movimientos inmigratorios hacia Las Rozas lo realizamos viendo las altas de inmigración que se han producido en el municipio en los últimos cinco años (abril de 1981-octubre de 1985). Con este material pretendiamos hacer un estudio que pusiera de manifiesto si existen algunas regularidades entre los movimientos intrametropolitanos, y si las hay, ver cuáles son las interrelaciones entre la movilidad y la estructura residencial del municipio.

Para ello y siguiendo las pautas que se han investigado en otros trabajos de este tipo y que se han quedado expuestas en el punto anterior, organizamos la información de las altas municipales de la siguiente manera:

1. Estudio de la procedencia.

En todas las altas viene el municipio de origen del nuevo residente, si procede de Madrid incluso viene en el distrito. Nosotros distinguimos:

- Procedentes de Madrid capital por distritos.

- Procedentes de los municipios del Area Metropolitana Sur.

- Procedentes de los municipios del Area Metropolitana Norte.

- Procedentes de los municipios del Area Metropolitana Este.

- Procedentes de los municipios del Area Metropolitana Oeste.

- Procedentes de los municipios de la Provincia de Madrid.

- Procedentes del resto de España.

- Procedentes del extranjero. 
2. Motivos o razones del cambio de residencia.

De la información de las altas obtuvimos los siguientes motivos:

- Nueva vivienda.

- Trabajo.

- Trabajo en Las Rozas.

- Accesibilidad al trabajo.

- Matrimonio.

- Divorcio.

- Familiar.

- Estudios.

3. Estudio del ciclo familiar.

El estudio del ciclo familiar lo estructuramos de la siguiente manera:

- Matrimonios jóvenes sin hijos.

- Matrimonios con hijos pequeños.

- Matrimonios con hijos mayores.

- Matrimonios mayores.

- Personas solas.

4. Características demográficas.

De la hoja de empadronamiento de cada nuevo residente, obtuvimos los siguientes grupos demográficos. 
- De 0 - 15 años.

- De 16 - 25 años.

- de 26 - 40 años.

- De 41 - 65 años.

- Más de 65 años.

5. Nivel educativo y socio-económico.

Características también obtenidas de la hoja padronal.

- Grado Medio.

- Título Superior.

- Trabajadores manuales.

- Técnicos.

- Personal directivo.

6. Año de llegada.

- 1981.

- 1982.

- 1983.

- 1984.

- 1985. 
Antes de analizar las características de la movilidad residencial en Las Rozas, vamos a comentar muy brevemente cómo han sido los movimientos migratorios en el municipio hasta el año 1981.

\section{RASGOS BASICOS DE LOS MOVIMIENTOS MIGRATORIOS EN LAS ROZAS}

A lo largo de la década de los sesenta, la inmigración se mantiene en unos valores moderados y estables. Sin embargo, ya en esta década se produce un cambio, ya que en el último lustro (1966-1970), la inmigración se convierte en el motor dinamizador de la demografia del municipio, papel que no ha dejado de jugar hasta el presente. En el pasado decenio (19701980 ), se acelera el ritmo de llegadas siendo el máximo (superior incluso a toda la inmigración anterior) en el quinquenio 1976-1980.

Si analizamos separadamente los años que componen este último período se observa una clara tendencia de aumento de las llegadas, aumento que como veremos, tendrá su continuidad en el período 1981-1985.

La rectificación anual del Padrón Municipal de Habitantes, que confecciona el Ayuntamiento, nos proporciona el número de inmigrantes y de emigrantes. Si bien las cifras son poco fiables, ya que no todas las personas se dan de alta al instalarse en el municipio, la comparación de los datos año a año es muy interesante.

El crecimiento año a año de los inmigrantes y el saldo migratorio concuerda con los datos anteriores, lo más sobresaliente del cuadro III es el hecho de que la emigración crece también, por lo menos desde 1976 en cifras absolutas. ¿Quiénes son esos inmigrantes? Posiblemente personas que utilizan Las Rozas como una etapa en su emigración a la capital u otros municipios del Area Metropolitana y también que emigran por no encontrar una vivienda que se acomode a sus posibilidades.

En efecto, según el estudio demográfico contenido en la Información Urbanística de $\mathrm{PGOU}{ }^{3}$ de Las Rozas el 12 por 100 de los emigrantes tiene

\footnotetext{
3 AVAnCE del PGou de Las Rozas (Información Urbanistica), pág. 97.
} 
como destino la capital, el 41 por 100 el resto del Area Metropolitana, el 15 por 100 la región centro (Avila, Segovia, Guadalajara, Cuenca y Toledo) y el 32 por 100 restante otras regiones del país. De todas maneras la emigración en el municipio de Las Rozas es muy pequeña comparada con la inmigración.

CUADRO II. RITMO DE LLEGADA DE LOS INMIGRANTES A LAS ROZAS

\begin{tabular}{|c|c|c|c|c|c|c|c|c|}
\hline \multirow[t]{2}{*}{$\begin{array}{l}\text { PERIODOS DE } \\
\text { TIEMPO }\end{array}$} & \multicolumn{2}{|c|}{ LAS ROZAS } & \multicolumn{2}{|c|}{ URBANIZACIONES } & \multicolumn{2}{|c|}{ LAS MATAS } & \multicolumn{2}{|c|}{$\begin{array}{c}\text { TOTAL } \\
\text { MUNICIPAL }\end{array}$} \\
\hline & VA & $\%$ & VA & $\%$ & VA & $\%$ & VA & $\%$ \\
\hline 1960 y antes & 324 & 6,9 & 204 & 7,7 & 264 & 15,1 & 792 & 8,7 \\
\hline $1961-1965 \ldots$ & 96 & 2,0 & 120 & 4,5 & 368 & 21,1 & 584 & 6,4 \\
\hline 1966-1970 & 328 & 6,9 & 112 & 4,2 & 140 & 8,0 & 580 & 6,4 \\
\hline $1971-1975$ & 924 & 19,6 & 576 & 21,8 & 276 & 15,8 & 1.776 & 19,5 \\
\hline $1976-1980 \ldots$ & 3.048 & 64,4 & 1.632 & 61,7 & 696 & 39,9 & 5.376 & 59,0 \\
\hline $1976 \ldots \ldots \ldots$ & 420 & 8,9 & 316 & 12,0 & 36 & 2,1 & 772 & 8,5 \\
\hline $1977 \ldots \ldots \ldots$ & 324 & 6,9 & 348 & 14,5 & 108 & 6,2 & 816 & 9,0 \\
\hline $1978 \ldots \ldots \ldots$ & 640 & 13,6 & 364 & 13,8 & 148 & 8,5 & 1.152 & 12,6 \\
\hline $1979 \ldots \ldots \ldots$ & 508 & 10,8 & 304 & 11,5 & 96 & 5,5 & 908 & 10,0 \\
\hline $1980 \ldots \ldots \ldots$ & 1.156 & 24,5 & 264 & 10,0 & 308 & 17,7 & 1.728 & 19,0 \\
\hline TOTAL .. & 4.720 & 100 & 2.644 & 100 & 1.744 & 100 & 9.108 & 100 \\
\hline
\end{tabular}

Fuente: Explotación manual del Padrón Municipal de habitantes de 1981. 
CUADRO III. SALDO MIGRATORIO. LAS ROZAS

\begin{tabular}{lccc}
\hline AÑO & INMIGRANTES & EMIGRANTES & SALDO \\
\hline 1975 & 267 & 143 & 124 \\
1976 & 327 & 49 & 278 \\
1977 & 720 & 158 & 562 \\
1978 & 1.237 & 164 & 1.073 \\
1979 & 1.548 & 188 & 1.360 \\
\hline
\end{tabular}

Fuente: Rectificación anual del Patrón municipal de habitantes y E.P.

En 1970 la tasa de inmigración era del 36,1 por 100 , del 48 por 100 en 1975 y de 67,4 por 100 en 1981. ¿Cuál es la procedencia de los inmigrantes? Vamos a comparar los datos que a este respecto aporta el Plan de Las Rozas con los obtenidos de la muestra del Padrón de 1981.

Madrid capital sigue siendo el gran aportador de población a Las Rozas, mientras que el Area Metropolitana sin la capital y el resto de la provincia, ha pasado de ocupar el tercer puesto por lugar de origen en 1975 al último puesto en 1981, incluso por debajo de los procedentes del extranjero. Pierde algo de peso la región centro mientras que aumentan considerablemente los inmigrantes del resto de las regiones. Los nacidos en el extranjero, que tengan otra nacionalidad o sean hijos de emigrantes nacidos fuera de España, conservan la proporción en valores parecidos.

Una vez tratadas a grandes rasgos las caracteristicas de los movimientos migratorios de Las Rozas, vamos a estudiar cómo han sido las inmigraciones más recientes. Como dijimos, para su estudio solamente contamos con la información - bastante interesante por otra parte- que nos proporcionan las altas de inmigración. No hemos podido contar con las bajas por emigración, ya que todavía no se habían elaborado para el quinquenio 19811985 , pero por la información obtenida oralmente a través de los encargados del Padrón municipal, sabemos que sigue las pautas del quinquenio 1976- 
1980, es decir, un ligerísimo aumento anual pero teniendo una importancia mínima en los movimientos migratorios del municipio.

Resumiendo lo expuesto anteriormente nos encontramos en 1981 con un municipio básicamente inmigratorio, cuya inmigración procede fundamentalmente de Madrid-Capital. La población que inmigra a Las Rozas busca principalmente una vivienda de calidad en la que residir.

Veamos ahora los rasgos de la inmigración más reciente.

Entre abril de 1981 y octubre de 1985 se han producido un total de 1.779 altas, que suponen 6.054 habitantes, lo que significa que si en 1981 la población municipal era de 13.405 habitantes, ésta ha aumentado por inmigración en los últimos cinco años en un 45,9 por 100. De las 1.779 altas,

CUADRO IV. ORIGEN DE LOS INMIGRANTES (\%) 1975 Y 1981

\begin{tabular}{|c|c|c|}
\hline ORIGEN & 1972 & 1981 \\
\hline MADRID CIUDAD & 41 & 42 \\
\hline AREA METROPOLITANA SIN MADRID & 7 & 2 \\
\hline RESTO PROVINCIA MADRID . .......... & 6 & 1,5 \\
\hline REGIÓN CENTRO $(x)$ & 12 & 10,5 \\
\hline RESTO ESPAÑA ... & 29 & 40 \\
\hline EXTRANJERO . & 5 & 4,5 \\
\hline TOTAL & 100 & 100 \\
\hline
\end{tabular}

(x) Avila, Segovia, Guadalajara, Cuenca y Toledo.

Fuente: PGOU. Las Rozas y EP. Padrón municipal de habitantes. Pág. 97. 
nosotros hemos tomado una muestra del 33 por 100 lo que supone el análisis de 593 altas y 2.098 habitantes.

\section{CUADRO V. EVOLUCION EN \% DE LAS ALTAS DE INMIGRACION DEL MUNICIPIO DE LAS ROZAS}

\begin{tabular}{ccc}
\hline AÑOS & $\%$ & \% ACUMULADO \\
\hline 1981 & 5,56 & 5,56 \\
1982 & 13,83 & 19,39 \\
1983 & 20,07 & 39,46 \\
1984 & 25,29 & 69,75 \\
1985 & 35,24 & 100 \\
\hline
\end{tabular}

Fuente: Padrón municipal y EP.

Aunque la inmigración al municipio en el último quinquenio ha descendido en algunos puntos (1976-1980, 59 por 100 y 1981-1985, 45,9 por 100), como vemos, la tendencia es a seguir aumentando año a año. En el caso de Las Rozas se sigue produciendo un intenso movimiento inmigratorio, posiblemente porque el tipo de vivienda de calidad que se construye en el municipio a precio asequible (una vivienda de los rasgos de la ofertada en Las Rozas en Madrid-capital multiplicaría por diez su precio), y por la consideración prestigiosa del sector en que se encuentra, asi como por la "relativa" buena comunicación que existe con Madrid a través de la N. VI, captan una demanda de clases medias profesionales que busca todo eso a la hora de elegir su lugar de residencia.

\section{PROCEDENCIA DE LOS NUEVOS RESIDENTES}

Como vemos en el cuadro VI, se ha producido un cierto cambio en la procedencia de los inmigrantes, aunque se sigue reforzando la corriente in- 
CUADRO VI. ORIGEN DE LOS INMIGRANTES (\%) 1981 Y 1985

\begin{tabular}{|c|c|c|}
\hline ORIGEN & 1981 & 1985 \\
\hline MADRID (ciudad) . & 42 & 51,2 \\
\hline AREA METROPOLITANA (sin Madrid) & 2 & 15,84 \\
\hline RESTO PROVINCIA DE MADRID .......... & 1,5 & 6,07 \\
\hline RESTO DE ESPAÑA & 55,5 & 23,94 \\
\hline EXTRANJERO .... & 4,5 & 3,6 \\
\hline TOTAL ........ & 100 & 100 \\
\hline
\end{tabular}

Fuente: Padrón municipal, altas de inmigración y EP.

migratoria desde Madrid-capital que se constituye, indudablemente, en el más importante aportador de población en Las Rozas. Sin embargo, son los habitantes procedentes de los municipios del Area Metropolitana los que han aumentado más comparativamente, pasando de una posición insignificante, a superar el 15,84 por 100 del total de nuevos residentes. Este hecho, en cierta Inanera viene a cumplir lo previsto en el «Informe sobre la población» del Consejo de Municipios (1980): «A partir de 1965 son los movimientos interiores los que van ganando protagonismo en la dinámica observada y, a corto plazo, no es aventurado afirmar que tal tendencia va a seguir siendo la determinante de los cambios en la estructura del poblamiento metropolitano, más que un incremento de población debido al aporte inmigratorio exógeno" 4 .

El frenazo de la inmigración hacia los grandes centros de población que ha ocurrido en los últimos años en España, ha supuesto que cada vez tenga, menos importancia el aporte exógeno de población (resto de España) mientras que son los flujos internos del área metropolitana los que aumentan junto con los procedentes de Madrid-capital.

\footnotetext{
${ }^{4}$ Consejo de municipios (1980): Informe sobre la Población. Madrid, pág. 100.
} 
A continuación vamos a matizar las características de los flujos provenientes de Madrid-capital y del Area Metropolitana. La población que tiene su procedencia en Madrid-capital la hemos clasificado por distritos.

Como podemos ver en el cuadro VII, son los cinco primeros distritos (Chamartín, Salamanca, Chamberí, Ciudad Lineal, y Moncloa) los que aportan casi el 60 por 100 de nuevos residentes; estos distritos, la mayoría de la zona del Ensanche o de crecimiento, son distritos que se caracterizan por su nivel socio-económico medio-alto, cumpliéndose así una de las características que se han puesto de manifiesto en los estudios de movilidad residencial, que es "la afinidad del vecindario" asi los habitantes de una zona de determinado estatus socioeconómico suelen ir a residir a áreas del mismo estatus.

En cuanto a la localización geográfica de la procedencia podemos observar que, prácticamente, los distritos de mayor aporte de población a Las Rozas están situados en el sector Norte de la ciudad, confirmándonos el hecho de que los cambios residenciales se suelen producir dentro del mismo sector. Sin embargo, pensamos que es sobre todo el parecido nivel socioeconómico lo que más influye a la hora de los traslados, lo que ocurre es que en Madrid se cumple que los distritos de mayor estatus están localizados - salvo excepciones- en el Norte de la ciudad, pudiendo adivinar una cierta sectorización en el desarrollo de las áreas de mayor nivel socioeconómico.

Por otra parte, vamos a ver cuáles son los principales rasgos de los flujos que tienen su origen en el resto de los municipios metropolitanos, que como hemos dicho han aumentado considerablemente en los últimos años.

De todos los inmigrantes procedentes de los municipios del Area Metropolitana, la mayoría tiene su origen en la zona oeste (Pozuelo, Majadahonda, Villanueva del Pardillo, Boadilla del Monte, Brunete), lo cual es lógico, puesto que Las Rozas se encuentra en este sector, y como hemos visto la movilidad residencial suele producirse fundamentalmente entre municipios del mismo sector. Lo más destacable de los datos expuestos en el cuadro VIII, es la importante corriente migratoria que proviene de los municipios del Sur del A. Metropolitana, las razones las podemos encontrar en que es ésta una de las zonas más pobladas del Area Metropolitana madrileña, y es la población de un cierto estatus que reside en este área la que se traslada a Las Rozas buscando un hábitat de mayor calidad y prestigio, que el que puede encontrar en sus municipios de origen. El resto de los municipios metropolitanos (Areas Este y Norte) apenas si tienen importancia como focos de origen de los nuevos habitantes de Las Rozas. 
CUADRO VII. INMIGRANTES PROCEDENTES DE MADRID CAPITAL POR DISTRITOS (\%) SEGUN ORIGEN DE IMPORTANCIA

\begin{tabular}{|c|c|c|}
\hline ORIGEN & $\%$ & $\%$ ACUMULADO \\
\hline CHAMARTIN & 17,11 & 17,11 \\
\hline SALAMANCA & 12,83 & 29,94 \\
\hline CHAMBERI & 12,17 & 42,11 \\
\hline CIUDAD LINEAL .... & 9,21 & 51,32 \\
\hline MONCLOA & 7,89 & 59,21 \\
\hline RETIRO $\ldots \ldots \ldots$. & 5,26 & 64,47 \\
\hline LATINA & 5,26 & 69,63 \\
\hline TETUAN & 4,27 & 74,00 \\
\hline ARGANZUELA $\ldots \ldots \ldots \ldots \ldots \ldots \ldots \ldots \ldots$ & 3,94 & 77,94 \\
\hline CENTRO & 3,62 & 81,56 \\
\hline MEDIODIA & 3,29 & 84,85 \\
\hline MORATALAZ & 3,29 & 88,14 \\
\hline CARABANCHEL $\ldots \ldots \ldots \ldots \ldots \ldots \ldots \ldots \ldots \ldots$ & 2,96 & 91,11 \\
\hline FUENCARRAL $\ldots \ldots \ldots \ldots \ldots \ldots \ldots \ldots \ldots$ & 2,63 & 93,73 \\
\hline VILLAVERDE $\ldots \ldots \ldots \ldots \ldots \ldots \ldots \ldots \ldots$ & 1,97 & 95,70 \\
\hline VALLECAS $\ldots \ldots \ldots \ldots \ldots \ldots \ldots \ldots \ldots \ldots \ldots \ldots \ldots \ldots$ & 1,64 & 97,34 \\
\hline$\ldots \ldots \ldots \ldots \ldots \ldots \ldots \ldots \ldots \ldots \ldots \ldots \ldots \ldots$ & 1,32 & 98,66 \\
\hline HORTALEZA $\ldots \ldots \ldots \ldots \ldots \ldots \ldots \ldots \ldots \ldots$ & 1,32 & 99,99 \\
\hline
\end{tabular}

Fuente: Padrón municipal, altas de inmigración y EP. 


\section{CUADRO VIII. INMIGRANTES PROCEDENTES DEL AREA METROPOLITANA (\%)}

\begin{tabular}{|c|c|c|}
\hline ORIGEN & $\%$ & $\%$ ACUMULADO \\
\hline A. METROPOLITANA OESTE & 49,38 & 49,38 \\
\hline A. METROPOLITANA SUR $\ldots \ldots \ldots \ldots \ldots \ldots \ldots$ & 40,45 & 89,83 \\
\hline A. METROPOLITANA ESTE & 7,86 & 97,69 \\
\hline A. METROPOLITANA NORTE $\ldots \ldots \ldots \ldots \ldots$ & 2,24 & 99,99 \\
\hline
\end{tabular}

Fuente: Padrón municipal, altas de imigración y EP.

Por último, en el resto de los lugares de origen de la inmigración hacia Las Rozas, tienen sobre todo algún peso la inmigración desde provincias, normalmente debido a traslados de trabajo; no se trata de personas que buscan empleo en Madrid, sino de ejecutivos o técnicos de empresas que se trasladan a Madrid y que eligen Las Rozas buscando una cierta calidad de hábitat. Finalmente, los residentes procedentes del extranjero apenas si tienen significación, de estos residentes, el 50 por 100 son europeos (destacan Alemania, Francia e Inglaterra), el 27,6 por 100 son americanos, el 21,4 por 100 de Africa y un 1 por 100 asiáticos (filipinas que se dedican al servicio doméstico).

\section{MOTIVOS DE LOS TRASLADOS}

En las altas por inmigración aparecen los motivos por los cuales se cambia de residencia, datos que nos ha parecido muy interesante a la hora del estudio de la movilidad residencial.

Como vemos en el cuadro IX, la principal razón del cambio de residencia es la adquisición de nueva vivienda, la gran movilidad que existe hacia Las Rozas en el momento presente, está en parte generada por la cantidad de viviendas en construcción y vacantes de calidad, que encuentran una demanda principalmente en el municipio de Madrid. Después del cambio de 


\section{CUADRO IX. MOTIVOS DEL CAMBIO DE RESIDENCIA (\%) POR ORDEN DE IMPORTANCIA}

\begin{tabular}{|c|c|c|}
\hline MOTIVO & $\%$ & $\%$ ACUMULADO \\
\hline Nueva vivienda & 51,26 & 51,26 \\
\hline Trabajo .................... & 22,93 & 74,19 \\
\hline Trabajo en Las Rozas ........ & 9,94 & 84,13 \\
\hline Matrimonio ........ & 5,23 & 89,36 \\
\hline Familiar & 4,38 & 93,74 \\
\hline Divorcio & 3,54 & 97,28 \\
\hline Accesibilidad al trabajo $\ldots . . .$. & 1,85 & 99,13 \\
\hline Estudios $\ldots \ldots \ldots \ldots \ldots \ldots \ldots$ & 0,84 & 99,99 \\
\hline
\end{tabular}

Fuente: Padrón municipal, altas de inmigración y EP.

vivienda, es el trabajo el segundo motivo en orden de importancia que se aduce para trasladarse a Las Rozas. En este caso, se trata de las personas que como hemos visto vienen de provincias a trabajar a Madrid y escogen Las Rozas como lugar de residencia. Ya que la oferta de trabajo en el municipio genera una corriente migratoria pequeña $(9,94 \%)$. Por lo que hemos podido ver en las altas, las personas que se trasladan a trabajar al municipio de Las Rozas lo hacen principalmente a oficinas bancarias o bien para trabajar en la construcción de viviendas. Son muy pocas las que se trasladaban a trabajar en las grandes empresas instaladas en el municipio (Kodak, Hewlett-Packard...) que suelen ser las que como motivo de cambio de residencia aducen «accesibilidad al trabajo».

Los cambios en la vida familiar (matrimonios, divorcios, ir a residir con un familiar) tienen un significado relativamente pequeño $(13,15 \%)$ aunque resulte que muchos de los que esgrimen cambios por adquisición de nueva 
vivienda también lo hagan por motivos familiares que a veces quedan ocultos. Sin embargo, en un municipio con rasgos demográficos jóvenes cabría esperar que el porcentaje de personas que por matrimonio trasladan allí su residencia fuese más alto. Esto hemos comprobado que no es así, porque como veremos en el siguiente apartado, no son los matrimonios recientes sino los matrimonios con hijos pequeños el segmento de población que más se traslada a residir al municipio. Resumiendo, en Las Rozas destacan los traslados voluntarios por adquisición de nueva vivienda. Esto es lógico que se produzca en un municipio donde lo que se va buscando es la calidad residencial. En la mayoría de los casos, la nueva vivienda suele ser la segunda que se adquiere a lo largo de la vida familiar. A través de estas líneas hemos dejado entrever la importancia que tiene en los traslados hacia Las Rozas, el momento del ciclo familiar, a continuación vamos a analizar esta característica.

\section{CICLO FAMILIAR Y RASGOS DEMOGRAFICOS DE LOS NUEVOS RESIDENTES}

El momento del ciclo familiar ha sido considerado como una de las motivaciones básicas para realizar los cambios de residencia hacia zonas suburbanas. Así, es la etapa de la vida familiar en la que se tienen los hijos pequeños, una etapa en la que se intenta buscar un hábitat con unas ciertas condiciones de vivienda, facilidad de colegios, espacios libres para juego, etcétera. Siendo los matrimonios con hijos pequeños los que suelen fijar su residencia en los suburbios. De todas maneras, en las ciudades americanas la existencia de una gran corriente hacia los suburbios ha supuesto que los segmentos de población que se dirigen hacia ellos estén conformados por grupos de personas más diversificadas. Así, recientemente se ha observado que no son mayoritariamente las familias con hijos pequeños las que se trasladan, sino que también presentan una gran movilidad otros grupos como personas solas, divorciados, matrimonios mayores sin hijos...

Para Las Rozas, hemos obtenido de las altas los siguientes datos sobre el ciclo familiar. 


\section{CUADRO X. ETAPA DEL CICLO FAMILIAR (\%) DE LOS NUEVOS RESIDENTES}

\begin{tabular}{|c|c|c|}
\hline ETAPA & $\%$ & $\%$ ACUMULADO \\
\hline Matrimonios con hijos pequeños & 57,35 & 57,71 \\
\hline Personas solas ... & 16,36 & 73,71 \\
\hline Matrimonios con hijos mayores ....... & 11,97 & 85,68 \\
\hline Matrimonios jóvenes sin hijos & 10,96 & 96,64 \\
\hline Matrimonios mayores $\ldots . .$. & 3,37 & 99,99 \\
\hline
\end{tabular}

Fuente: Padrón municipal, altas de inmigración y EP.

Como vemos, a Las Rozas, municipio suburbano de reciente formación, son principalmente los matrimonios con hijos pequeños los que se trasladan a vivir. El segundo grupo de población con un porcentaje significativamente menor, está constituido por personas solas. Por lo que hemos podido comprobar, que se trata de profesionales-medios que buscan en el municipio una vivienda de calidad y un entorno social agradable; también en este grupo se encuentran personas divorciadas y personas mayores que por motivos familiares, normalmente ir a residir con un pariente, se trasladan al municipio. Es de destacar que los matrimonios jóvenes sin hijos ocupan la penúltima posición de los grupos familiares que establecen su residencia en Las Rozas, la razón la podemos buscar en la relativa carestía de la vivienda, que a un matrimonio recién establecido le es difícil afrontar.

Así pues, con respecto al momento de la vida familiar, la inmigración hacia Las Rozas, tiene unos rasgos clarísimos, son los matrimonios con hijos (sobre todo con hijos pequeños) los que alli se trasladan. Ya que uno de los mayores atractivos del municipio son los espacios libres, los servicios que ofrecen las viviendas (jardines, piscinas, complejos deportivos etc...), que se adecuan a las necesidades familiares sobre todo en la primera etapa de la vida familiar cuando los niños son pequeños.

Las características demográficas de la población que se traslada al municipio viene a corroborar lo anteriormente expuesto. 
Son fundamentalmente los grupos de población correspondientes a matrimonios jóvenes (26-40 años) y a los hijos pequeños (0-15 años) los que se trasladan al municipio.

\section{CUADRO XI. RASGOS DEMOGRAFICOS DE LOS} NUEVOS RESIDENTES (\%)

\begin{tabular}{|c|c|}
\hline GRUPOS DE EDAD & $\%$ \\
\hline $0-15$ & 37,68 \\
\hline $16-25 \ldots \ldots$ & 10,46 \\
\hline $26-40 \ldots \ldots \ldots \ldots \ldots \ldots \ldots \ldots$ & 39,54 \\
\hline $41-65 \ldots \ldots \ldots \ldots \ldots \ldots \ldots \ldots$ & 10,06 \\
\hline Más de $65 \ldots \ldots \ldots \ldots \ldots \ldots$ & 2,22 \\
\hline
\end{tabular}

Fuente: Padrón municipal, altas de inmigración y EP. 


\section{BIBLIOGRAFIA}

CLARKE, S. D. (1975): La sociedad suburbana, IEAL. Madrid.

COPLACO (1975): Estructura residencial y mercado de la vivienda, Análisis de problemas y oportunidades. Madrid.

DolCE, P. C. (1976): Suburbia: The American Dream and Dilemma, Anchor Books, N. York.

Fernández DuRÁn, R. (1980): "La necesidad de desplazarse y la estructura urbana y social: el caso de Madrid". Ciudad y Territorio n. $^{\circ} 2$ pp. $33-48$.

JOHNSON, J. M. (1974): Suburban Growth. Wiley. Londres.

Kramer, J. (1972): North American Suburbs. The Glendessary Press. Berkeley.

PITChard, R. (1976): «Residential mobility and the housing market» en English city since the industrial revolution. Cambridge University Press.

Santos Preciado, J. M. (1985): «El modelo de diferenciación residencial en el sector suroeste del A. M. Madrileña". Tesis doctoral. Universidad Complutense de Madrid. 